\title{
Feedforward Inhibition Determines the Angular Tuning of Vibrissal Responses in the Principal Trigeminal Nucleus
}

\author{
Marie-Andrée Bellavance, Maxime Demers, and Martin Deschênes \\ Centre de Recherche Université Laval Robert-Giffard, Québec City, Québec G1J 2R3, Canada
}

Trigeminal neurons that relay vibrissal messages to the thalamus receive input from first-order afferents that are tuned to different directions of whisker motion. This raises the question of how directional tuning is maintained in central relay stations of the whisker system. In the present study we performed a detailed analysis of the angular tuning properties of cells in the principal trigeminal nucleus of the rat. We found that stimulus direction systematically influences response latency, so that the degree of directional tuning and the preferred deflection angle computed with first-spike latency yielded results nearly similar to those obtained with spike counts. Furthermore, we found that inhibition sharpens directional selectivity, and that pharmacological blockade of inhibition markedly decreases the angular tuning of cellular responses. These results indicate that the angular tuning of cells in the first relay station of the vibrissal system is determined by fast feedforward inhibition, which shapes excitatory inputs at the very beginning of synaptic integration.

\section{Introduction}

Primary afferents and neurons in trigeminal sensory nuclei, thalamus and cortex of the rodent whisker system are sensitive to the direction of whisker deflection (Simons and Carvell, 1989; Lichtenstein et al., 1990; Bruno et al., 2003; Minnery and Simons, 2003; Minnery et al., 2003; Wilent and Contreras, 2005; Andermann and Moore, 2006). It is generally agreed that the directional selectivity of primary afferents depends on the spatial distribution of receptors in vibrissal follicle (Ebara et al., 2002), but the circuitry and mechanisms that maintain directional selectivity in central relay stations remain poorly understood. Approximately 150-200 trigeminal ganglion cells innervate each whisker follicle in rodents (Welker and Van der Loos, 1986; Crissman et al., 1991). Regardless of their response properties, these cells form ladder-like projection patterns in trigeminal nuclei, consisting of several puffs of terminations distributed at regular interval (150$200 \mu \mathrm{m}$ ) (Hayashi, 1980; Henderson and Jacquin, 1995; Shortland et al., 1996). The discontinuous arbors from each of the fibers innervating a single whisker interdigitate to produce a rostrocaudally continuous column of terminations that is coextensive with the barrelette corresponding to the same vibrissa. Because relay cells in the principal trigeminal nucleus (PrV) extend dendrites over 300-500 $\mu \mathrm{m}$ within a barrelette (Arends and Jacquin, 1993; Veinante and Deschênes, 1999), they should collect input from a number of primary vibrissa afferents that are tuned to different directions. Thence the question arises of how angular tuning is maintained in the PrV-to-barrel cortex pathway.

Directional selectivity is also a feature of many neurons in the visual and auditory systems, where experimental and modeling

\footnotetext{
Received Sept. 28, 2009; revised Nov. 4, 2009; accepted Dec. 5, 2009.

This research was supported by Canadian Institutes of Health Research Grant MT-5877 (M. Deschênes).

Correspondence should be addressed to Martin Deschênes at the above address. E-mail: martin.deschenes@ crulrg.ulaval.ca.

DOI:10.1523/JNEUROSCI.4805-09.2010

Copyright $\odot 2010$ the authors $\quad 0270-6474 / 10 / 301057-07 \$ 15.00 / 0$
}

studies have shown that inhibition plays a key role in sharpening direction tuning by limiting the ability of weaker inputs to evoke action potentials (Delorme, 2003; Monier et al., 2003; Wehr and Zador, 2003). The PrV contains local circuit inhibitory cells and receives inhibitory input from the interpolar trigeminal nucleus (SpVi) (Avendaño et al., 2005; Furuta et al., 2008), but the contribution of inhibition in sharpening directional selectivity of vibrissal responses has never been investigated. In the present study we provide a comprehensive assessment of the mechanisms underlying directional tuning in the $\mathrm{PrV}$, and we show that pharmacological blockade of inhibition markedly reduces the directional selectivity of PrV cells.

\section{Materials and Methods}

Experiments were conducted in accordance with federally prescribed animal care and use guidelines. The Ethical Committee for Animal Use in Research (Laval University) approved all experimental protocols.

Recording of $\operatorname{PrV}$ cells. Experiments were performed in 8 male rats (250-300 g; Sprague Dawley) under ketamine $(75 \mathrm{mg} / \mathrm{kg}$, xylazine 5 $\mathrm{mg} / \mathrm{kg}$ ) anesthesia. The left facial nerve was cut, and the rat was placed in a stereotaxic apparatus. The animal breathed freely, and body temperature was maintained at $37.5^{\circ} \mathrm{C}$ with a heating pad controlled thermostatically. Throughout the experiment, a deep level of anesthesia was maintained (stage III-3) (Friedberg et al., 1999) by additional doses of anesthetics $(20 \mathrm{mg} / \mathrm{kg}$ ketamine plus $0.3 \mathrm{mg} / \mathrm{kg}$ xylazine, i.m.) given as needed to abolish reflex to sharp pinch of the hindlimbs.

A hole ( $\sim 2 \mathrm{~mm}$ in diameter) was drilled over the left PrV (frontal plane 9.3 behind the bregma, $2.8 \mathrm{~mm}$ lateral to the midline; Paxinos and Watson, 1998), and the dura was resected with the curve tip of a 27-gauge needle, care being taken not to damage the posterior blood sinus. The micropipette was lowered vertically, and single PrV units were recorded extracellularly with micropipettes (tip diameter, $0.5-1 \mu \mathrm{m}$ ) filled with a solution of potassium acetate $(0.5 \mathrm{M})$. Whisker-responsive units were recorded at depths of $6.5-8 \mathrm{~mm}$ below the pia. Once a PrV cell was encountered, its receptive field was mapped. The micropipette was further advanced to record large action potentials $(4-8 \mathrm{mV})$. Directional tuning was assessed, and we tested for the presence of inhibition by inducing background discharges with juxtacellular current injection 
(1-5 nA). Signals were amplified, bandpass filtered $(100 \mathrm{~Hz}-3 \mathrm{kHz})$, and sampled at $10 \mathrm{kHz}$. When current injection resulted in cell impalement (4 cases) the gain was reduced, and the high pass filter was set at $1 \mathrm{~Hz}$.

Whisker stimulation. Vibrissae were cut at $5 \mathrm{~mm}$ from the skin, and we assessed the receptive field size of single units by deflecting individual vibrissae with a hand-held probe under a dissecting microscope. An audiomonitor and a computer display were used to monitor the responses. Then, the tip of the vibrissa was inserted into the groove of a beveled straw attached to a ceramic bimorph bender (Physik Instrumente). The vibrissa was pushed in a given direction at stimulus onset, but returned passively at a neutral position at stimulus offset. To ensure that the axis of the stimulator was aligned with that of the hair shaft, we restricted our study to cells that responded to whiskers of rows C-E and arcs 2-5. Ramp-and-hold waveforms (rise/fall times, $8 \mathrm{~ms}$; total duration, $70 \mathrm{~ms}$; amplitude, $0.2-0.6 \mathrm{~mm}$; interstimulus interval, $1.2 \mathrm{~s}$ ) were used to deflect vibrissae from their resting position in eight directions spanning $360^{\circ}$ (e.g., in $45^{\circ}$ increments relative to the horizontal alignment of the vibrissa rows). Stimuli were repeated 25 times, the probe was rotated by $45^{\circ}$, and the sequence was repeated. As measured with a photodiode, resonance frequency was $200 \mathrm{~Hz}$ (amplitude, $\sim 20 \mu \mathrm{m}$ for the first period of the largest deflection).

Brainstem lesions. Before recording in $\operatorname{PrV}$, the spinal trigeminal complex was acutely lesioned in 2 rats by passing direct current $(3 \mathrm{~mA}, 3 \mathrm{~s})$ through a tungsten electrode (shaft diameter, $200 \mu \mathrm{m}$; tip diameter, 50 $\mu \mathrm{m}$; deinsulated over $1 \mathrm{~mm}$ ). Lesion was made at the frontal plane 11.5 $\mathrm{mm}$ behind bregma (stereotaxic coordinates from the atlas of Paxinos and Watson, 1998), and current was passed at multiple depths to fully destroy ascending projections to the PrV. At the end of the recording session, animals were perfused under deep anesthesia with saline followed by a solution of $4 \%$ paraformaldehyde plus $0.2 \%$ glutaraldehyde in phosphate buffer $(0.1 \mathrm{M}, \mathrm{pH}$ 7.4). In each lesioned rat the extent of the lesion was assessed on cytochrome oxidase-stained frontal sections of the brainstem.

Local injection of inhibitory receptor antagonists. The angular tuning of $\mathrm{PrV}$ neurons was assessed in 2 rats after local injection of a saline solution $(0.5-1 \mu \mathrm{l})$ containing gabazine (10 mM; Tocris Bioscience) and strychnine (5 mm; Tocris Bioscience). The PrV was first localized by unit recording, and drugs were injected at the same location by pressure (pipette diameter: $20 \mu \mathrm{m}$; Nanoliter 2000, WPI). Recordings were performed near the injection site within 90 min after the injection.

Data analysis. Spike events elicited by vibrissa deflection were collected in peristimulus time histograms (PSTHs) of 20 responses with $1 \mathrm{~ms}$ bin width. The magnitude of vibrissal responses was estimated as the mean number of spikes per deflection within poststimulus time windows of 10 and $20 \mathrm{~ms}$. The mean firing rate was measured in a time window of 3-20 $\mathrm{ms}$ after stimulus onset, the $3 \mathrm{~ms}$ offset representing the minimum conduction time between the follicle and PrV. Peak frequency of ON responses was estimated from the shortest interspike interval within a time window of 3-10 ms after stimulus onset. The modal latency of the first spike occurring within a time window of $10 \mathrm{~ms}$ after stimulus onset was used to estimate first-spike latency (bin size, $200 \mu \mathrm{s}$ ). We chose the modal instead of the mean latency because of its insensitivity to extreme values. The latency of inhibition was estimated as the first of 5 consecutive bins $(200 \mu \mathrm{s})$ after stimulus onset where the mean number of counts/bin was significantly smaller than the mean number of counts/bin in the $50 \mathrm{~ms}$ prestimulus time window ( $p<0.01$; one-tailed $t$ test).

Directional tuning in the vibrissal system is usually assessed by using spike counts as a dependent variable (Simons and Carvell, 1989; Lichtenstein et al., 1990; Bruno et al., 2003; Minnery and Simons, 2003; Minnery et al., 2003). However, recent studies indicated that precise spike timing in ventral posterior medial nucleus (VPM) can also convey a remarkable amount of information (Montemurro et al., 2007; Petersen et al., 2008). We thus used the mean number of spikes per deflection and first-spike latency to build polar plots of angular preference. Our analyses did not consider plateau and OFF responses.

For each cell, a direction-selectivity index, $D$, was calculated as a measure of the directional tuning (Taylor and Vaney, 2002). Using normalized values of the mean number of spikes per deflection, $D$ was defined as $D=\Sigma v_{\mathrm{i}} / \Sigma r_{\mathrm{i}}$ (i.e., $D=$ vector sum/scalar sum), where $v_{\mathrm{i}}$ are vector mag- nitudes pointing in the direction of the stimulus and having length, $r_{\mathrm{i}}$, equal to the number of spikes recorded during that stimulus. $D$ can range from 0 , when the responses are equal in all stimulus directions, to 1 , when a response is obtained only for a single stimulus direction. Thus, values for $D$ approaching 1 indicate asymmetric responses over a small range of angles and therefore sharper directional tuning. To estimate to what degree computed $D$ values resulted from a small-sample bias, the responses of each neuron were randomly redistributed over the eight angles, and $D$ values were averaged over 10,000 shuffles. We then subtracted the resulting $D$ value from the original $D$ value for each neuron, to obtain an unbiased index of angular tuning.

Direction-selectivity index was also computed using first-spike latency. First-spike latency at all responsive angles were normalized with respect to the shortest latency (i.e., latency minimum/latency), which yielded polar graphs of angular tuning that were compared with those obtained with spike counts. Data were analyzed with the Neuroexplorer (Plexon) Excel (Microsoft), Oriana (Kovac Computing Service) and Matlab (MathWorks) software. Results are reported as mean \pm SD.

\section{Results}

The PrV comprises two classes of projection cells: small-sized barrelette cells that project exclusively to the VPM barreloids, and large-sized interbarrelette cells that project to several downstream stations (superior colliculus, thalamus, cerebellum, etc; Bruce et al., 1987; Bennett-Clarke et al., 1992; Arends and Jacquin, 1993; Veinante and Deschênes, 1999). Although both classes of cells respond to multiple vibrissae in lightly anesthetized animals (Minnery and Simons, 2003), the receptive field of barrelette cells shrinks to a single vibrissa under deep anesthesia. The present study focuses on barrelette cells, which were identified by their monowhisker receptive field as assessed with a piezoelectric stimulator. These cells had low level of spontaneous activity $(<0.1 \mathrm{~Hz})$, but responded briskly to vibrissa deflection.

\section{Stimulus-evoked activity}

We recorded and analyzed the response properties of $41 \mathrm{PrV}$ cells in 4 normal rats. Of this sample, $37 \%$ of the cells were classified as slowly adapting units (SA) on the basis that they fired, on average, $>2$ action potentials per deflection at the most effective angle during the stimulus plateau (time interval: 20-60 ms after stimulus onset). We chose this rather conservative criterion to ensure a reliable identification of SA units. The other cells were considered rapidly adapting (RA) by default. Vibrissal responses of a representative SA unit are shown in Figure $1 \mathrm{~A}$.

Several measures were used to estimate response magnitude at the maximally responsive angle. The mean firing rate across all cells in the time interval of 3-20 ms after stimulus onset was 162.2 $\pm 67.4 \mathrm{~Hz}(\mathrm{SA}, 210.8 \pm 47.7 \mathrm{~Hz} ; \mathrm{RA}, 133.3 \pm 60.9 \mathrm{~Hz})$, and the mean firing rate during the plateau response of SA units was $111.5 \pm 32.8 \mathrm{~Hz}$. Peak instantaneous frequency computed between $3-10 \mathrm{~ms}$ after stimulus onset averaged up to $819.7 \pm 233.7$ $\mathrm{Hz}$ for all cells $(\mathrm{SA}, 856.5 \pm 185.1 \mathrm{~Hz}$; RA, $796.2 \pm 261.0 \mathrm{~Hz}$; highest frequency, $1.2 \mathrm{kHz}$ ). These high firing rates are the more remarkable as $\mathrm{ON}$ responses of $\mathrm{PrV}$ units often consisted of 2-5 spikes emitted in a frequency range of $0.8-1 \mathrm{kHz}$ (Fig. $1 \mathrm{~B}$ ).

\section{Directional tuning}

Consistent with previous reports, the majority of PrV cells exhibited a fair degree of directional sensitivity, with $81 \%$ of the cells being nonresponsive to at least one direction (null direction; i.e., no evoked spike within a time window of $20 \mathrm{~ms}$ after stimulus onset). The average number of null directions per cell amounts to $2.83 \pm 1.46$ for the whole population; no significant difference 


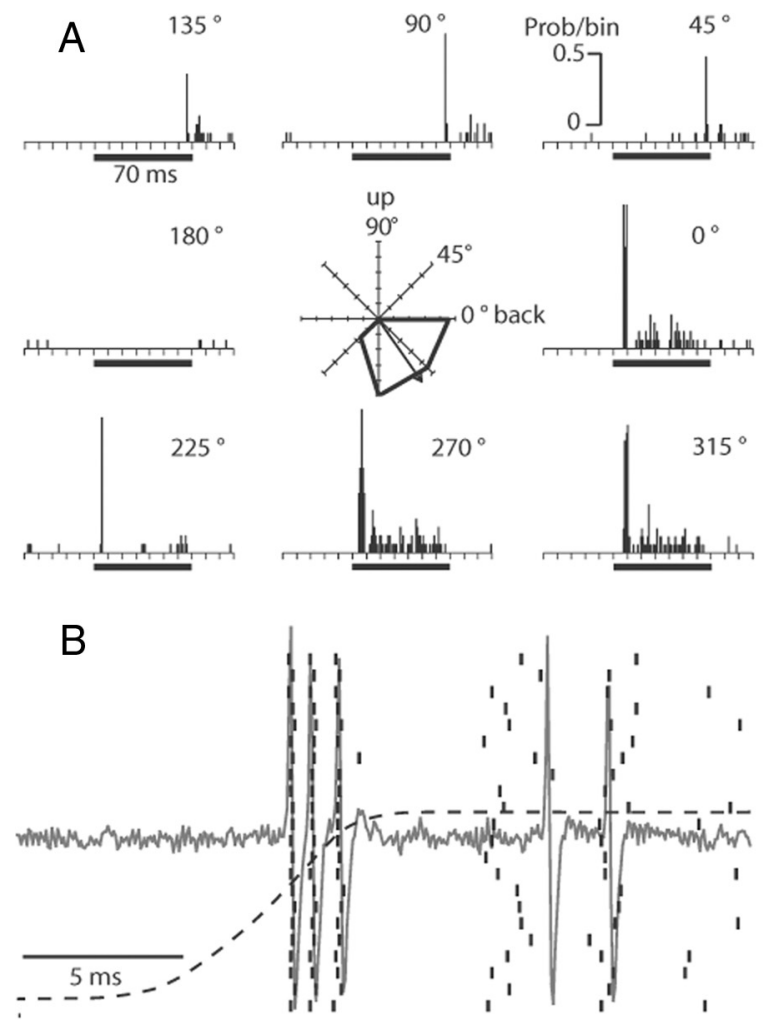

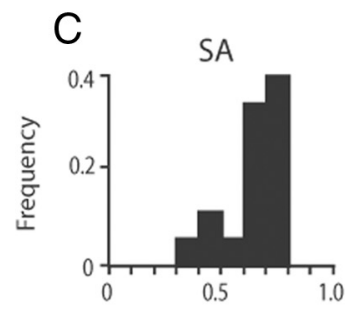

RA

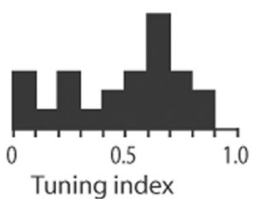

RA

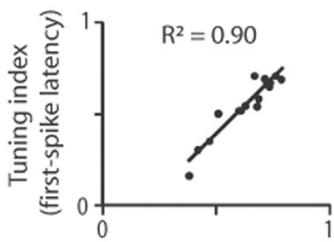

E

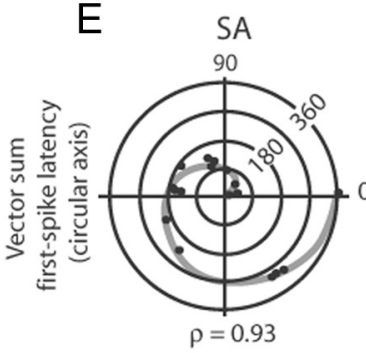

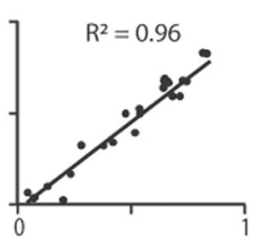

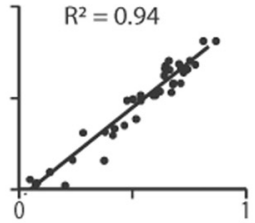

Tuning index (spike/stimulus)

RA

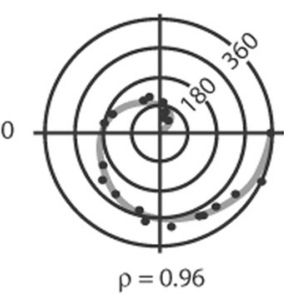

$R A \& S A$

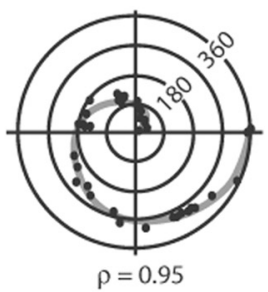

$R A \& S A$

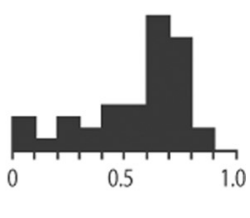

$R A \& S A$

Figure 1. Response of PrV cells to controlled whisker deflection. PSTHs and the polar plot in $\boldsymbol{A}$ show responses of a SA unit to directional deflection of whisker D3. Normalized number of spikes per deflection within a time window of $10 \mathrm{~ms}$ after stimulus onset was used to build the polar plot; the arrow is the vector sum of angular responses. Traces and raster display in $\boldsymbol{B}$ show $0 \mathrm{~N}$ responses of a representative SA unit; note the very high frequency of action potentials ( $\sim 1 \mathrm{kHz})$; dotted line, stimulus waveform. $C$, Distribution of tuning index across a population of 16 SA and $27 \mathrm{RA}$ units. Scatter plots in $\boldsymbol{D}$ show correlation between tuning indices computed from normalized values of spike counts per stimulus (10 ms time window) and first-spike latency (regression lines: $S A, y=$ $1.21 x-0.22 ; \mathrm{RA}, y=1.02 x-0.06$; RA and $\mathrm{SA}, y=1.02 x-0.07$ ). $\boldsymbol{E}$, Circular-circular correlograms between preferred deflection angle computed as the vector sum of normalized values of spike counts per stimulus ( $10 \mathrm{~ms}$ time window) and first-spike latency. The gray line is the curve of a perfect correlation. In these circular plots, the vector sum derived from first-spike latency is represented on the circular axis, and the vector sum derived from spike counts is represented on the radial axis.

was found between SA and RA units $(3.24 \pm 0.90$ vs $2.54 \pm 1.72$; $p=0.14 ; t$ test $)$.

Across the whole population of PrV cells the mean amount of angular tuning ( $D$ value, see Materials and Methods) was $0.55 \pm$ 0.22 , being slightly higher for SA units than for RA units $\left(D_{\mathrm{SA}}=\right.$ $0.63 \pm 0.12 ; D_{\mathrm{RA}}=0.49 \pm 0.25 ; p=0.08$; Mann-Whitney; Fig. $1 C)$. The cell in Figure $1 A$, for example, had a $D$ value of 0.73 , and supplemental Figure S1 (available at www.jneurosci.org as supplemental material) provides polar plots with the associated $D$ values for each of the cells in our sample. Whether computed with poststimulus time windows of 10 or $20 \mathrm{~ms}, D$ values were not significantly different ( $p=0.24$; paired sign test).

To estimate to what extent $D$ values resulted from a sampling bias, the responses of each neuron were randomly redistributed over the eight angles, and $D$ values were averaged over 10,000 shuffles. The mean $D$ value obtained after shuffling was subtracted from the original $D$ value for each neuron, yielding an unbiased measure of angular tuning. Across the whole population of neurons, the difference between mean $D$ values of the original and shuffled data was highly significant $(0.55 \pm 0.22 \mathrm{vs}$ $0.30 \pm 0.10 ; p<0.0001$; paired sign test), indicating that original results are very robust to bias correction. This was confirmed by regression analysis, which revealed a strong positive association between bias-corrected and original $D$ values $\left(R^{2}=0.972\right.$; supplemental Fig. S2, available at www.jneurosci.org as supplemental material).
Across the whole population of PrV cells, the first spike at the maximally responsive angle occurred at a mean latency of $5.1 \pm$ $1.2 \mathrm{~ms}$ (SA, $4.9 \pm 1.0$; RA, $5.4 \pm 1.2 ; p=0.14 ; t$ test). Angular tuning index estimated from normalized values of first-spike la-

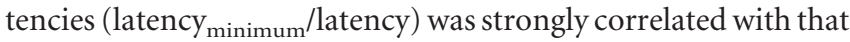
estimated from spike counts $\left(R^{2}=0.94\right.$; Fig. $\left.1 D\right)$. A very strong correlation was also found between the preferred deflection angle computed as the vector sum of spike counts ( $10 \mathrm{~ms}$ poststimulus time window) and that estimated with the normalized values of first-spike latency $(r=0.95 ; p<0.05$; circular-circular correlation; Fig. $1 E$ ). A similar degree of correlation was obtained when a poststimulus time window of $20 \mathrm{~ms}$ was used to estimate spike counts $(r=0.93 ; p<0.05$; circular-circular correlation). For this test we excluded three nontuned RA units $(D<0.1$; cells 39, 40 and 41 in supplemental Fig. S1, available at www.jneurosci.org as supplemental material) because the best direction estimated from either spike counts or first-spike latencies was meaningless (a small difference in spike counts could induce large unpredictable shift of the best direction. Figure 2 shows the absolute angular difference between preferred directions estimated by the normalized values of first-spike latency and spike counts versus the directional tuning index of PrV cells.

In sum, our analysis show that stimulus direction systematically influences response latency, so that the degree of directional tuning and the preferred deflection angle computed with first- 
spike latency yielded results nearly similar to those obtained with spike counts.

\section{Inhibition shapes the angular tuning of PrV neurons}

We next examined whether inhibition shapes the angular tuning of PrV cells. This test was performed in 35 cells (cells 1-35 in supplemental Fig. S1, available at www.jneurosci.org as supplemental material) by deflecting the vibrissa in a null direction during sustained discharges induced by juxtacellular current injection. A clear inhibition of background discharges was observed in all cells, which was preceded by an excitation in $37 \%$ of the cases. When the early excitation was absent (22 cases), the mean onset latency of inhibition was $5.9 \pm 1.1 \mathrm{~ms}$. The PSTHs of Figure $3, A$ and $B$, show examples of pure inhibitory responses observed in a single cell and at population level. Intracellular recordings ( 4 cells) also provided direct evidence for direction-sensitive inhibition. The cell shown in Figure $3 C$ responded with a spike on the crest of an EPSP at a deflection angle of $135^{\circ}$, whereas the responses consisted of an IPSP at a deflection angle of $45^{\circ}$ (note the opposite pattern of excitation/inhibition at stimulus offset).

\section{Origin of inhibition-interpolaris lesion}

Prior studies have shown that the PrV contains GABAergic and glycinergic neurons (Avendaño, et al., 2005), and that it also receives profuse inhibitory input from the SpVi (Furuta et al., 2008). We first examined how lesion of the interpolaris input affected the angular tuning of PrV neurons. We recorded and analyzed 39 cells in 2 lesioned rats. These cells displayed low spontaneous activity $(<0.2$ $\mathrm{Hz}$ ) and, according to the criterion used in normal rats, a larger proportion of cells (56\%) were classified as SA units. The mean firing rate at the maximally responsive angle during the poststimulus time window of 3-20 ms was significantly higher than in normal rats $(251.1 \pm 100.0$ $\mathrm{Hz}$ for all cells; $p<10^{-5}$; $t$ test; $321.9 \pm$ $60.6 \mathrm{~Hz}$ for SA units, and $159.5 \pm 56.0 \mathrm{~Hz}$ for RA units). Peak instantaneous frequency estimated between 3 and $10 \mathrm{~ms}$ $\mathrm{Hz}$ for all cells $(953.5 \pm 104.6 \mathrm{~Hz}$ for SA units, and 904.1 $\pm 128.4 \mathrm{~Hz}$ for RA units).

As a population, $\mathrm{PrV}$ cells in lesioned rats had direction tuning index significantly lower than in normal rats $(D=0.32 \pm 0.24$; $p<0.0003$; Mann-Whitney; $D_{\mathrm{sa}}=0.28 \pm 0.22 ; D_{\mathrm{ra}}=0.37 \pm$ 0.27; Fig. 4A). Yet, 17 cells (44\%; 8 SA units and 9 RA units) still failed to respond to at least one direction. Although the amount and duration of inhibition were markedly diminished in lesioned rats, it was not totally eliminated. A short-lasting period of inhibition ( $\sim 5 \mathrm{~ms})$ was still detected in 8 cells where no excitation preceded inhibition (Fig. $4 B$, arrow).

Histological controls confirmed that lesions completely destroyed the descending limb of the trigeminal tract and the caudal part of the oralis subnucleus, but they never extended rostrally beyond the emergence of the facial nerve (supplemental Fig. S2, available at www.jneurosci.org as supplemental material). Thus, the main effect of lesioning connections between the SpVi and the after stimulus onset rose to $932.0 \pm 116.6$

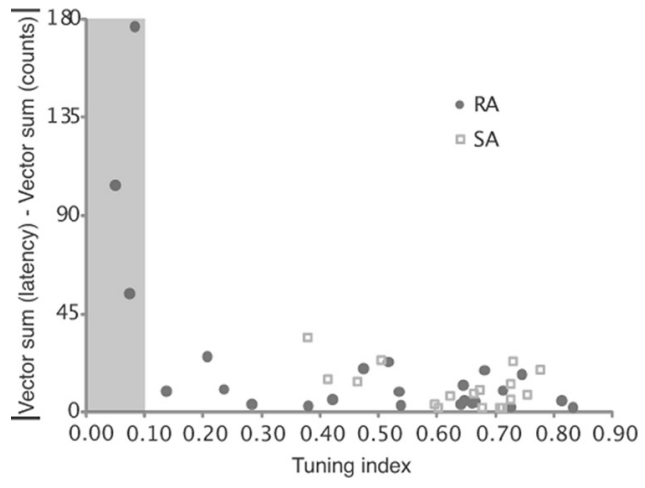

Figure 2. Consistency of latency and spike-count directional tuning in PrV. The absolute angular difference between preferred directions estimated by the normalized values of firstspike latency and spike-counts is plotted as a function of the directional tuning index of PrV cells. Note the important difference for the least tuned cells (gray column). These three cells correspond to cells 39, 40 and 41 in supplemental Figure $\mathrm{S1}$, available at www.jneurosci.org as supplemental material; they were excluded from the circular-circular correlation analysis (see Fig. 1E).
Figure 3. Inhibition shapes the angular tuning of PrV neurons. Single cell PSTH in $\boldsymbol{A}$ and population PSTH in $\boldsymbol{B}$ show the suppression of background discharges induced by current injection (bar labeled DC under the PSTHs) when whiskers were deflected in the least effective direction. Intracellular recordings in $\mathbf{C}$ show a cell that responded with a spike on the crest of an EPSP at a deflection angle of $135^{\circ}$, and with an IPSP at a deflection angle of $45^{\circ}$ (5 superimposed traces; note the opposite pattern of excitation/inhibition at stimulus offset).
Prob/bin $0.03-$

DC


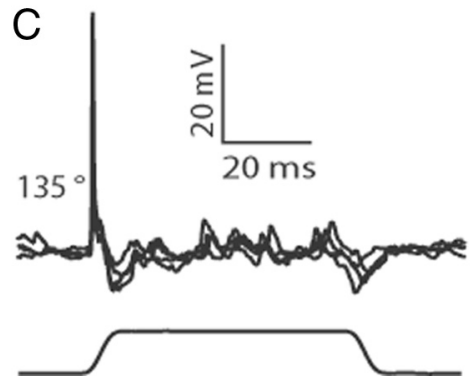

show the
deflected
pattern of at a

$\operatorname{PrV}$ was to reduce whisker-evoked inhibition, which broadened the angular tuning of PrV cells.

\section{Origin of inhibition - effect of GABAergic and glycinergic antagonists}

Lesion of SpVi projections, although extensive, did not totally suppress whisker-evoked inhibition in the PrV, suggesting that residual inhibition was mediated by local circuit inhibitory neurons within the PrV (Avendaño et al., 2005; Furuta et al., 2008). We examined how pharmacological blockade of inhibition by local injection of gabazine (a GABAa receptor antagonist) and strychnine (a glycinergic receptor antagonist) affects the angular tuning of $\operatorname{PrV}$ cells.

The vibrissal responses of $21 \mathrm{PrV}$ cells (2 rats) were recorded within 90 min after an intra-PrV injection of inhibitory receptor 

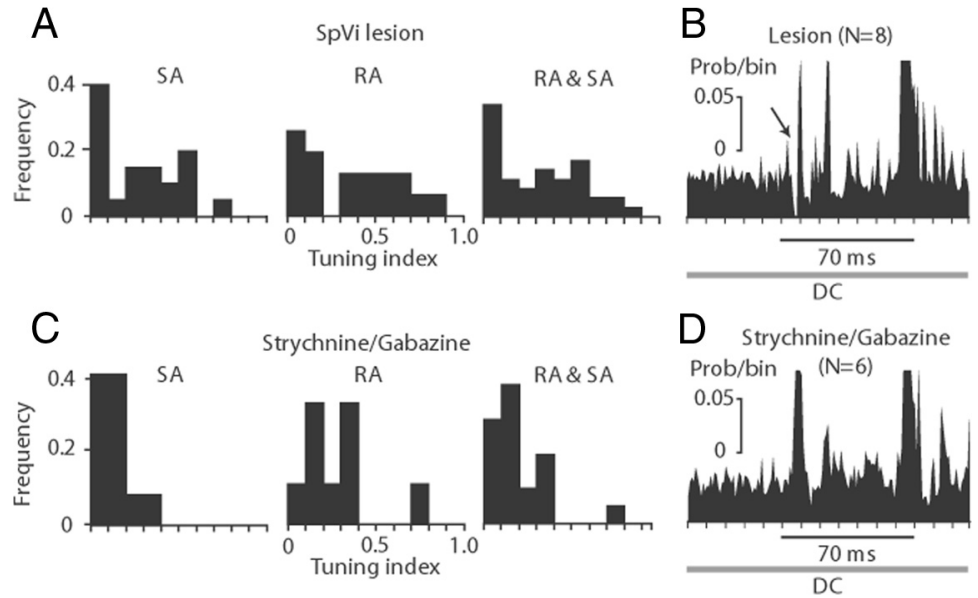

Figure 4. Blockade of inhibition reduces the angular tuning of PrV cells. Lesion of the inhibitory connections between the SpVi and the PrV significantly reduces the tuning index of PrV cells in comparison with normal rats $(p<0.0003$ for the combined populations of $S A$ and RA units; compare histograms in $A$ with histograms of Fig. 1C). A short-lasting suppression of background discharges induced by current injection (bar labeled DC under the PSTHs) was still present when whiskers were deflected in the least effective direction (arrow in $\boldsymbol{B}$ ). This population PSTH was built from the responses of 8 cells where no excitation preceded inhibition. C, When inhibition was fully blocked by local injection of gabazine and strychnine, tuning indices were further reduced with respect to normal rats ( $p<0.0001)$, and background discharges were no more suppressed when whiskers were deflected in the least effective direction (D). Note that in $\boldsymbol{B}$ and $\boldsymbol{D}$, the magnitude of $\mathrm{ON}$ and $\mathrm{OFF}$ responses was cropped. test; SA units, $340.8 \pm 158.2 \mathrm{~Hz}$; RA units, $241.4 \pm 75.5 \mathrm{~Hz}$ ). Peak instantaneous frequency at response onset, which was already high in lesioned rats, neither increased significantly (all cells best direction, 921.2 $\pm 137.2 \mathrm{~Hz}$; SA units, $946.6 \pm 137.7 \mathrm{~Hz}$; RA units, $887.2 \pm 137.9 \mathrm{~Hz}$ ). The main effects on directional selectivity were to further reduce the degree of angular tuning $(D=$ $0.19 \pm 0.15 ; p<0.0001$ with respect to normal rats; Mann-Whitney; $D_{\text {sa }}=$ $0.12 \pm 0.08 ; D_{\text {ra }}=0.28 \pm 0.18$; Fig. $4 C$ ), and decrease the proportion of neurons with at least one null direction (3 cells; i.e., $14 \%$ ). Responses of 6 cells to the least effective direction were examined under background activity induced by current injection. As shown in the population PSTH of Figure $4 D$, inhibition was absent in these neurons, and cell depolarization revealed the presence of excitatory inputs that remained subthreshold at rest.

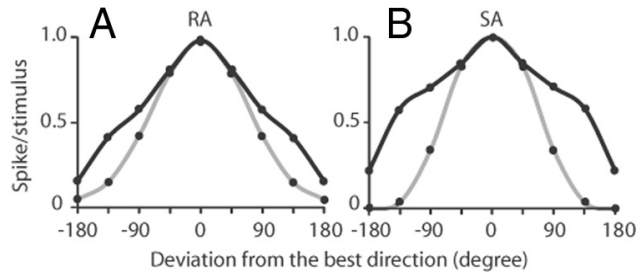

Figure 5. Broadening of the angular tuning domain of PrV cells after blockade of inhibition by gabazine and strychnine. Graphs show the average attenuation of response magnitude as a function of the angular distance from the best direction for RA $(\boldsymbol{A})$ and $S A(B)$ units (gray line, control rats; black line, rats injected with gabazine and strychnine).

Table 1. Properties of vibrissal responses in the PrV

\begin{tabular}{|c|c|c|c|}
\hline Response properties & Normal rats & $\begin{array}{l}\text { SpVi-lesioned } \\
\text { rats }\end{array}$ & $\begin{array}{l}\text { Gabazine } \\
\text { and strychnine }\end{array}$ \\
\hline SA units (\%) & 37 & 56 & 63 \\
\hline RA units (\%) & 63 & 44 & 37 \\
\hline Mean frequency & $162.2 \pm 67.4$ & $251.1 \pm 100^{* * *}$ & $298 \pm 136.3^{* * *(\#)}$ \\
\hline \multicolumn{4}{|l|}{ ON response $(\mathrm{Hz})^{a}$} \\
\hline Peak frequency & $819.7 \pm 233.7$ & $932.0 \pm 116.6^{* *}$ & $921.2 \pm 137.2^{* * *(\#)}$ \\
\hline \multicolumn{4}{|l|}{$0 \mathrm{~N}$ response $(\mathrm{Hz})^{b}$} \\
\hline First-spike latency $(\mathrm{ms})^{c}$ & $5.1 \pm 1.2$ & $4.7 \pm 1.1^{*}$ & $4.9 \pm 1.0^{\#(\#)}$ \\
\hline Onset latency of inhibition (ms) & $5.9 \pm 1.1$ & $5.6 \pm 1.0^{\#}$ & - \\
\hline Tuning index (counts/stimulus) $)^{b}$ & $0.55 \pm 0.22$ & $0.32 \pm 0.24^{* * *}$ & $0.19 \pm 0.15^{* * *(\#)}$ \\
\hline Number of null directions/cell & $2.83 \pm 1.46$ & $1.08 \pm 1.46^{* * *}$ & $0.34 \pm 0.89^{* * *(*)}$ \\
\hline \multicolumn{4}{|c|}{ In a time window of 20 ms after stimulus onset; best direction. } \\
\hline \multicolumn{4}{|c|}{${ }^{b}$ In a time window of $10 \mathrm{~ms}$ after stimulus onset; best direction. } \\
\hline \multicolumn{4}{|l|}{ Best direction. } \\
\hline \multicolumn{4}{|c|}{$\begin{array}{l}{ }^{*} p<0.05 ;{ }^{* *} p<0.01 ;{ }^{* * *} p<0.001 ;{ }^{* *} p>0.05 \text {, Significance level with respect to normal rats. } \\
\left(*_{0}\right)<0.05 \text {. }\left(\text { (H) }_{p}>0.05 \text {. Significancevel with respect tolesioned rats. }\right.\end{array}$} \\
\hline
\end{tabular}

antagonists. Drug injection did not affect the spontaneous activity of monowhisker PrV cells, which remained low $(<0.5$ $\mathrm{Hz}$ ), but the proportion of cells classified as SA units rose to $63 \%$. The mean firing rate at the maximally responsive angle increased in comparison with lesioned rats, but the difference was not statistically significant $(298.2 \pm 136.3 \mathrm{~Hz} ; p=0.13 ; t$

\section{Angular domain of inhibition}

To determine the angular domain over which direction-sensitive inputs are preferentially suppressed by inhibition, we constructed rotated, population polar plots. Individual normalized polar plots were rotated so that maximally responsive angles were aligned in a common direction, and an average response at each angle was calculated based on the entire population. Then, the resulting polar plot was pivoted about the axis passing through the maximally responsive angle to obtain a mirror image plot that was averaged with the original population polar plot. This yielded a symmetrical graph reflecting the average attenuation of the responses as a function of the angular distance from the best direction. As shown in Figure 5, inhibition principally depressed responses to deflection angles that deviated by $>45^{\circ}$ from the best direction, the more so for SA than for RA units. Difference in response magnitude that persists under gabazine/ strychnine is likely attributable to difference in the synaptic impact of primary afferents that are tuned to different directions.

\section{Summary of results}

Table 1 provides a summary of the quantitative changes in response properties produced by SpVi lesion and drug injection. The most salient features consist of an increase in the proportion of cells classified as SA units, an increase in the magnitude of ON responses, a marked reduction in the degree of angular tuning and in the number of null directions. Furthermore, our analysis revealed that first-spike latency is as reliable as spike counts to quantify angular tuning in the PrV.

\section{Discussion}

The present study focused on monowhisker cells, which constitute the majority of $\mathrm{PrV}$ neurons that project to the thalamic barreloids (Veinante and Deschênes, 1999; Minnery and Simons, 2003). Our sample may include some local circuit cells but, at least for the response properties examined, there is no indication that these cells might differ from thalamic-projecting monowhisker cells. Yet, we examined only responses to passive whisker deflection in deeply anesthetized rats. During tactile exploration 
in behaving animals top-down mechanisms should affect the way these two classes of neurons process vibrissal inputs.

Experiments were performed under deep anesthesia, which reduces the contribution of intersubnuclear excitatory inputs to receptive field synthesis in PrV (Minnery and Simons, 2003; Timofeeva et al., 2003). Comparison of our results with those obtained in fentanyl sedated rats suggests that, apart from reducing receptive field size, and increasing the number of null directions, the deep plane of anesthesia little affected response latency and the degree of directional tuning of PrV neurons.

\section{Rate code versus latency code}

Directional preference in sensory systems is usually assessed by the number of spikes emitted within a fixed time window after stimulus onset. In a recent study in humans, Johansson and Birznieks (2004) reported the first direct evidence that the relative timing of the first impulses in the median nerve reliably conveys information about the direction of force applied to the fingertip. Our results also show that stimulus direction systematically influences first-spike latency in the PrV, so that first-spike time yields directional tuning curves similar to those obtained with spike counts. Because the PrV is the first processing stage of vibrissal information, spike timing likely plays a key role as well in encoding directional preference in thalamus and cortex. Yet, to maintain time coding at thalamic level, vibrissal responses in $\mathrm{PrV}$ must be well matched to the biophysical properties of synaptic transmission at lemniscal synapses. One of the factors that limit the efficacy of synaptic transmission in the VPM is adaptation, a form of synaptic depression that is particularly severe in alert animals (Castro-Alamancos, 2002; Swadlow et al., 2005). Thus, a code that relies on first-spike latency alone might not be effective enough to overcome synaptic adaptation. Lemniscal EPSPs are characterized by very fast rise times $(\sim 400 \mu \mathrm{s})$, and half-decay times of 2-3 ms, which allows very little time for EPSP summation (Deschênes et al., 2003). The remarkable ability of PrV cells to generate short-latency spike bursts of exceptionally high frequency $(800-1000 \mathrm{~Hz})$ appears optimal for preserving the direction-dependent time code of vibrissal messages at thalamic level. Given the low degree of input convergence at trigeminothalamic synapses (a VPM cell receives input, on average, from one PrV neuron; Castro-Alamancos, 2002; Deschênes et al., 2003; Arsenault and Zhang, 2006), this mechanism explains why, despite adaptation, directional properties of a stimulus are for the most part faithfully preserved across the trigeminothalamic synapse (Minnery et al., 2003; Khatri and Simons., 2007; Khatri et al., 2009).

Given the high degree of synaptic convergence at cortical level (Bruno and Sakmann, 2006), the angular preference of barrel neurons strongly depends on synchronous firing of barreloid neurons (Pinto et al., 1996, 2000). Accordingly, first-spike synchrony among thalamic cells activated by the same stimulus angle was identified as the main factor that determines the angular tuning of barrel neurons (Temereanca et al., 2008). This is supported by intracellular recording which showed that as direction diverges from the preferred direction vibrissa-evoked EPSPs in barrel neurons decrease in amplitude and increase in onset latency (Wilent and Contreras, 2005).

\section{Decoding spike latency: reference signals}

Previous experimental and theoretical studies of vibrissal responses have consistently shown that precise spike timing conveys more information than spike count alone (Panzeri et al., 2001; Foffani and Moxon, 2004; Arabzadeh et al., 2006; Montemurro et al., 2007;
Petersen et al., 2008). Yet, the use of a spike latency code requires a reference signal for decoding its meaning.

In laboratory situations, stimulus onset can serve as an obvious reference signal. Several internally generated events have been proposed as reference signals to decode the timing of spikes in naturalistic situations (VanRullen et al., 2005; Chase and Young, 2007). One of these, are central events associated with motor commands that control whisker motion, or ocular saccades in the visual system. As discussed by VanRullen et al. (2005): "By parsing the input stream into discrete events, these reference signals would turn each successive sample into a new stimulus with its own 'onset' time followed by its proper sequence of first spikes". A model that has received the greatest theoretical and empirical examination is a phase-locked loop, a circuit optimal for temporal decoding that relies on the comparison of an input signal with a centrally generated reference signal (Ahissar, 1998; Ahissar and Kleinfeld, 2003). Although a number of predictions derived from this model have received experimental support, the existence of a central time reference associated with whisker motion still awaits confirmation in behaving animals. Another intriguing possibility is that the brain does not use an external reference signal to extract stimulus onset, but derives this information from latency differences in large populations of cortical neurons with broad receptive fields (Foffani et al., 2008).

\section{Fast feedforward inhibition and angular tuning}

As suggested by the anatomical organization of the terminal fields of primary afferents in $\operatorname{PrV}$, our results show that individual barrelette cells receive input from ganglion cells that respond to virtually all directions of whisker deflection. This is demonstrated by the fact that, after inhibition was blocked, the number of null directions dramatically decreased, and spikes were evoked at all deflection angles when cells were depolarized.

Results also clearly demonstrate that fast feedforward inhibition shapes excitatory inputs at the very beginning of synaptic integration. Because inhibitory interneurons are likely activated at short latency by primary afferents that best respond at any given direction, the degree of inhibition depends on the order of arrival of incoming spikes. Thus, substantial excitation precedes inhibition only for a range of directions close to the preferred one, so that deflections in that range are more likely to generate spikes.

The fact that inhibition plays a key role in sharpening angular tuning suggests that the degree of angular tuning of a PrV cell may vary depending on the excitability of inhibitory interneurons or the efficacy of inhibitory synaptic transmission. Thus, in theory, central mechanisms that control the excitability of interneurons could adjust the gain of synaptic transmission, and therefore modify the angular tuning of cellular responses.

\section{Angular tuning and whisker torsion}

When whiskers protract, they rotate around their longitudinal axis (Knutsen et al., 2008). Depending on protraction amplitude, the torsion angle can reach up to $\sim 90^{\circ}$, which implies that whisker contact at different torsion angles is associated with different activity profiles across directionally tuned primary afferents. Accordingly, the angular tuning of a PrV cell could shift by as much as $90^{\circ}$ with respect to head-centered coordinates. Interestingly, this amount of shift remains within an angular domain over which PrV cells are best tuned, inhibition preferentially suppressing responses that deviate by more than $\pm 45^{\circ}$ from the best direction. In thalamus and cortex also, the majority of the tuning profiles are poorly selective to deflection angles of $<90^{\circ}$ (Simons and Carvell, 1989; Minnery et al., 2003; Timofeeva et al., 2003). 
For the moment, there is no indication that rodents use the direction of vibrissa motion as a sensory cue for precise object location or fine-grained texture discrimination. If they do, however, there should exist a mechanism that transforms vibrissacentered into head-centered coordinates to detect the origin of a stimulus at different torsion angles.

\section{References}

Ahissar E (1998) Temporal-code to rate-code conversion by neuronal phase-locked loops. Neural Comput 10:597-650.

Ahissar E, Kleinfeld D (2003) Closed-loop neuronal computations: focus on vibrissa somatosensation in rat. Cereb Cortex 13:53-62.

Andermann ML, Moore CI (2006) A somatotopic map of vibrissa motion direction within a barrel column. Nat Neurosci 9:543-551.

Arabzadeh E, Panzeri S, Diamond ME (2006) Deciphering the spike train of a sensory neuron: counts and temporal patterns in the rat whisker pathway. J Neurosci 26:9216-9226.

Arends JJ, Jacquin MF (1993) Lucifer Yellow staining in fixed brain slices: optimal methods and compatibility with somatotopic markers in neonatal brain. J Neurosci Methods 50:321-339.

Arsenault D, Zhang ZW (2006) Developmental remodelling of the lemniscal synapse in the ventral basal thalamus of the mouse. J Physiol 573:121-132.

Avendaño C, Machín R, Bermejo PE, Lagares A (2005) Neuron numbers in the sensory trigeminal nuclei of the rat: a GABA- and glycine-immunocytochemical and stereological analysis. J Comp Neurol 493:538-553.

Bennett-Clarke CA, Chiaia NL, Jacquin MF, Rhoades RW (1992) Parvalbumin and calbindin immunocytochemistry reveal functionally distinct cell groups and vibrissa-related patterns in the trigeminal brainstem complex of the adult rat. J Comp Neurol 320:323-338.

Bruce LL, McHaffie JG, Stein BE (1987) The organization of trigeminotectal and trigeminothalamic neurons in rodents: a double-labeling study with fluorescent dyes. J Comp Neurol 262:315-330.

Bruno RM, Sakmann B (2006) Cortex is driven by weak but synchronously active thalamocortical synapses. Science 312:1622-1627.

Bruno RM, Khatri V, Land PW, Simons DJ (2003) Thalamocortical angular tuning domains within individual barrels of rat somatosensory cortex. J Neurosci 23:9565-9574.

Castro-Alamancos MA (2002) Properties of primary sensory (lemniscal) synapses in the ventrobasal thalamus and the relay of high-frequency sensory inputs. J Neurophysiol 87:946-953.

Chase SM, Young ED (2007) First-spike latency information in single neurons increases when referenced to population onset. Proc Natl Acad Sci U S A 104:5175-5180.

Crissman RS, Warden RJ, Siciliano DA, Klein BG, Renehan WE, Jacquin MF, Rhoades RW (1991) Numbers of axons innervating mystacial vibrissa follicles in newborn and adult rats. Somatosens Mot Res 8:103-109.

Delorme A (2003) Early cortical orientation selectivity: how fast inhibition decodes the order of spike latencies. J Comput Neurosci 15:357-365.

Deschênes M, Timofeeva E, Lavallée P (2003) The relay of high-frequency sensory signals in the whisker-to-barreloid pathway. J Neurosci 23:67786787.

Ebara S, Kumamoto K, Matsuura T, Mazurkiewicz JE, Rice FL (2002) Similarities and differences in the innervation of mystacial vibrissal folliclesinus complexes in the rat and cat: a confocal microscopic study. J Comp Neurol 449:103-119.

Foffani G, Moxon KA (2004) PSTH-based classification of sensory stimuli using ensembles of single neurons. J Neurosci Methods 135:107-120.

Foffani G, Chapin JK, Moxon KA (2008) Computational role of large receptive fields in the primary somatosensory cortex. J Neurophysiol 100:268-280.

Friedberg MH, Lee SM, Ebner FF (1999) Modulation of receptive field properties of thalamic somatosensory neurons by the depth of anesthesia. J Neurophysiol 81:2243-2252.

Furuta T, Timofeeva E, Nakamura K, Okamoto-Furuta K, Togo M, Kaneko T, Deschênes M (2008) Inhibitory gating of vibrissal inputs in the brainstem. J Neurosci 28:1789-1797.

Hayashi H (1980) Distributions of vibrissae afferent fibers collaterals in the trigeminal nuclei as revealed by intra-axonal injection of horseradish peroxidase. Brain Res 183:442-446.

Henderson TA, Jacquin MF (1995) What makes subcortical barrels? In: Ce- rebral cortex. The barrel cortex of rodents (Jones EG, Diamond IT, eds), pp 123-188. New York: Plenum.

Johansson RS, Birznieks I (2004) First spikes in ensembles of human tactile afferents code complex spatial fingertip events. Nat Neurosci 7:170-177.

Khatri V, Simons DJ (2007) Angularly nonspecific response suppression in rat barrel cortex. Cereb Cortex 17:599-609.

Khatri V, Bruno RM, Simons DJ (2009) Stimulus-specific and stimulusnonspecific firing synchrony and its modulation by sensory adaptation in the whisker-to-barrel pathway. J Neurophysiol 101:2328-2338.

Knutsen PM, Biess A, Ahissar E (2008) Vibrissal kinematics in 3D: tight coupling of azimuth, elevation, and torsion across different whisking modes. Neuron 59:35-42.

Lee S, Carvell GE, Simons DJ (2008) Motor modulation of afferent somatosensory circuits. Nat Neurosci 11:1430-1438.

Lichtenstein SH, Carvell GE, Simons DJ (1990) Responses of rat trigeminal ganglion neurons to movements of vibrissae in different directions. Somatosens Mot Res 7:47-65.

Minnery BS, Simons DJ (2003) Response properties of whisker-associated trigeminothalamic neurons in rat nucleus principalis. J Neurophysiol $89: 40-56$.

Minnery BS, Bruno RM, Simons DJ (2003) Response transformation and receptive-field synthesis in the lemniscal trigeminothalamic circuit. J Neurophysiol 90:1556-1570.

Monier C, Chavane F, Baudot P, Graham LJ, Frégnac Y (2003) Orientation and direction selectivity of synaptic inputs in visual cortical neurons: a diversity of combinations produces spike tuning. Neuron 37:663-680.

Montemurro MA, Panzeri S, Maravall M, Alenda A, Bale MR, Brambilla M, Petersen RS (2007) Role of precise spike timing in coding of dynamic vibrissa stimuli in somatosensory thalamus. J Neurophysiol 98:1871-1882.

Panzeri S, Rolls ET, Battaglia F, Lavis R (2001) Speed of feedforward and recurrent processing in multilayer networks of integrate-and-fire neurons. Network 12:423-440.

Paxinos G, Watson C (1998) The rat brain in stereotaxic coordinates, Ed 4. London: Academic.

Petersen RS, Brambilla M, Bale MR, Alenda A, Panzeri S, Montemurro MA, Maravall M (2008) Diverse and temporally precise kinetic feature selectivity in the VPm thalamic nucleus. Neuron 60:890-903.

Pinto DJ, Brumberg JC, Simons DJ, Ermentrout GB (1996) A quantitative population model of whisker barrels: re-examining the Wilson-Cowan equations. J Comput Neurosci 3:247-264.

Pinto DJ, Brumberg JC, Simons DJ (2000) Circuit dynamics and coding strategies in rodent somatosensory cortex. J Neurophysiol 83:1158-1166.

Shortland PJ, Demaro JA, Shang F, Waite PM, Jacquin MF (1996) Peripheral and central predictors of whisker afferent morphology in the rat brainstem. J Comp Neurol 375:481-501.

Simons DJ, Carvell GE (1989) Thalamocortical response transformation in the rat vibrissa/barrel system. J Neurophysiol 61:311-330.

Swadlow HA, Bezdudnaya T, Gusev AG (2005) Spike timing and synaptic dynamics at the awake thalamocortical synapse. Prog Brain Res 149:91-105.

Taylor WR, Vaney DI (2002) Diverse synaptic mechanisms generate direction selectivity in the rabbit retina. J Neurosci 22:7712-7720.

Temereanca S, Brown EN, Simons DJ (2008) Rapid changes in thalamic firing synchrony during repetitive whisker stimulation. J Neurosci 28:11153-11164.

Timofeeva E, Mérette C, Emond C, Lavallée P, Deschênes M (2003) A map of angular tuning preference in thalamic barreloids. J Neurosci 23:10717-10723.

VanRullen R, Guyonneau R, Thorpe SJ (2005) Spike times make sense. Trends Neurosci 28:1-4.

Veinante P, Deschênes M (1999) Single- and multi-whisker channels in the ascending projections from the principal trigeminal nucleus in the rat. J Neurosci 19:5085-5095.

Wehr M, Zador AM (2003) Balanced inhibition underlies tuning and sharpens spike timing in auditory cortex. Nature 426:442-446.

Welker E, Van der Loos H (1986) Quantitative correlation between barrelfield size and the sensory innervation of the whiskerpad: a comparative study in six strains of mice bred for different patterns of mystacial vibrissae. J Neurosci 6:3355-3373.

Wilent WB, Contreras D (2005) Dynamics of excitation and inhibition underlying stimulus selectivity in rat somatosensory cortex. Nat Neurosci 8:1364-1370. 